\title{
Accuracy of Monocular Two-Dimensional Pose Estimation Compared With a Reference Standard for Kinematic Multiview Analysis: Validation Study
}

Oskar Stamm, MSc; Anika Heimann-Steinert, Dr rer medic

Geriatrics Research Group, Charité - Universitätsmedizin Berlin, corporate member of Freie Universität Berlin, Humboldt-Universität zu Berlin, and Berlin Institute of Health, Berlin, Germany

\section{Corresponding Author:}

Oskar Stamm, MSc

Geriatrics Research Group

Charité - Universitätsmedizin Berlin, corporate member of Freie Universität Berlin, Humboldt-Universität zu Berlin, and Berlin Institute of Health

Reinickendorfer Straße 61

Berlin, D-13347

Germany

Phone: 4930450553784

Email: oskar.stamm@charite.de

\section{Abstract}

Background: Expensive optoelectronic systems, considered the gold standard, require a laboratory environment and the attachment of markers, and they are therefore rarely used in everyday clinical practice. Two-dimensional (2D) human pose estimations for clinical purposes allow kinematic analyses to be carried out via a camera-based smartphone app. Since clinical specialists highly depend on the validity of information, there is a need to evaluate the accuracy of 2D pose estimation apps.

Objective: The aim of the study was to investigate the accuracy of the 2D pose estimation of a mobility analysis app (Lindera-v2), using the PanopticStudio Toolbox data set as a reference standard. The study aimed to assess the differences in joint angles obtained by $2 \mathrm{D}$ video information generated with the Lindera-v2 algorithm and the reference standard. The results can provide an important assessment of the adequacy of the app for clinical use.

Methods: To evaluate the accuracy of the Lindera-v2 algorithm, 10 video sequences were analyzed. Accuracy was evaluated by assessing a total of 30,000 data pairs for each joint (10 joints in total), comparing the angle data obtained from the Lindera-v2 algorithm with those of the reference standard. The mean differences of the angles were calculated for each joint, and a comparison was made between the estimated values and the reference standard values. Furthermore, the mean absolute error (MAE), root mean square error, and symmetric mean absolute percentage error of the 2D angles were calculated. Agreement between the 2 measurement methods was calculated using the intraclass correlation coefficient (ICC[A,2]). A cross-correlation was calculated for the time series to verify whether there was a temporal shift in the data.

Results: The mean difference of the Lindera-v2 data in the right hip was the closest to the reference standard, with a mean value difference of $-0.05^{\circ}\left(\mathrm{SD} 6.06^{\circ}\right)$. The greatest difference in comparison with the baseline was found in the neck, with a measurement of $-3.07^{\circ}\left(\mathrm{SD} 6.43^{\circ}\right)$. The MAE of the angle measurement closest to the baseline was observed in the pelvis $\left(1.40^{\circ}, \mathrm{SD} 1.48^{\circ}\right)$. In contrast, the largest MAE was observed in the right shoulder $\left(6.48^{\circ}, \mathrm{SD} 8.43^{\circ}\right)$. The medians of all acquired joints ranged in difference from $0.19^{\circ}$ to $3.17^{\circ}$ compared with the reference standard. The ICC values ranged from 0.951 (95\% CI 0.914-0.969) in the neck to 0.997 (95\% CI 0.997-0.997) in the left elbow joint. The cross-correlation showed that the Lindera-v2 algorithm had no temporal lag.

Conclusions: The results of the study indicate that a 2D pose estimation by means of a smartphone app can have excellent agreement compared with a validated reference standard. An assessment of kinematic variables can be performed with the analyzed algorithm, showing only minimal deviations compared with data from a massive multiview system.

(JMIR Mhealth Uhealth 2020;8(12):e19608) doi: 10.2196/19608

\section{KEYWORDS}

2D human pose estimation; motion capturing; kinematics; clinical practice; mobility; smartphone app; analysis 


\section{Introduction}

Traditional movement assessments, although carried out by experienced physicians, physiotherapists, and occupational therapists, can contain inaccuracies due to subjectivity, despite the clinicians' expertise. In contrast, quantitative motion measurements by motion capture systems are a valuable tool in scientific and clinical motion analysis and offer a highly accurate and reliable way of capturing kinematic data. Quantitative analyses can be used, for example, to monitor the progress of therapies and objectively evaluate the effectiveness of specific interventions. Motion capture systems are used in sports, biomechanics, and rehabilitation, and they focus on gait analysis, injury prevention, and performance improvement [1]. However, these systems are rarely used in everyday clinical practice.

There are many optoelectronic motion capture systems based on markers (eg, Vicon [Vicon Motion Systems], Motion Analysis [Motion Analysis Corp], Optitrack [NaturalPoint Inc], and Qualisys [Qualisys AB]). These systems are often regarded in the literature as the gold standard for motion capture [2]. Today, low- to high-speed experiments show that positioning errors can even be assumed in the case of measurements less than $2 \mathrm{~mm}$ [3]. Nevertheless, optoelectronic systems require a restricted area, such as a laboratory environment, and the attachment of markers [4], which can also be a potential source of measurement error in these systems due to skin movement artifacts [5]. Furthermore, these systems are proving to be very costly. The aforementioned factors are detrimental to the systems' practicability and everyday use in a clinical context.

Inertial sensor measurement systems can be used as a low-cost alternative. However, an inertial sensor measurement system cannot determine global position when used as a stand-alone system (by itself), although as a fusion system, in combination with a rigid body model such as the Perception Neuron (Noitom Ltd), a position in space can still be identified [2]. Nevertheless, a major disadvantage remains in clinical practice, as users must attach numerous sensors to a patient's body. Since clinical processes are usually efficiency driven and the application of several sensors is too time-consuming in most cases due to time constraints during treatment, this also contributes to the low frequency of use. Recently, studies have examined markerless and body sensor-less image-processing systems. Depth-sensing camera systems, such as the Kinect (Microsoft Corp), Intel RealSense (Intel Corp), or Zed (Stereolabs Inc) sensors, have proven to be a cost-effective solution with acceptable accuracy for some use cases [6-11]. Another low-cost motion capture method in image processing is pose estimation, which involves transformation of two-dimensional (2D) images into three-dimensional (3D) objects, for example, by using deep convolutional neural networks of monocular images [12-15], such as images on a mobile device [16]. Research into depth estimation from a monocular image is still in its infancy in the field of computer vision and is proving to be difficult in some cases, as slight inaccuracies in estimation can lead to very different depth estimates [17,18]. Major limitations include false pose estimates due to the target person moving outside the

image boundaries [19] or the pose being disturbed by objects such as shadows [15].

A 2D skeleton detector enables calculation of specific joint angles for assessment and feedback in sport and rehabilitation settings. The use of 2D human pose estimations for clinical purposes, such as the Lindera-v2 app or the motion-tracking coach on the Kaia health app [20], allows mobility analyses to be carried out using, for example, a mobile device. Pose and movement analyses via a smartphone save time for medical staff, who can use the time gained as treatment time or for patient consultations. Furthermore, such a measurement of mobility represents a more objective method of measurement compared with traditional assessments, which are based on a subjective assessment. Since in clinical practice, specialist staff highly depend on the validity of information, there is a need to validate the methodology of the Lindera-v2 measuring method. In order to achieve a performance level comparable with the gold standard motion capture systems, this study aimed to evaluate the accuracy of the Lindera-v2 2D pose estimation algorithm, using the PanopticStudio Toolbox (Carnegie Mellon University) $[21,22]$ as a reference standard.

\section{Methods}

\section{Data Collection}

For the accuracy evaluation, 10 video sequences were generated from Panoptic Studio 3D PointCloud (data set 171204_pose1-6) $[21,22]$. Data shared for research purposes from Carnegie Mellon University were used as the reference standard. The data set included video sequences from 480 video graphics array (VGA) cameras, 31 high-definition (HD) cameras, and 10 Kinect cameras, as well as arrays of 2D poses of key points of body part locations, showing the range of motion of the joints. The videos were split so that only one actor appeared in each video. Subsequently, 10 videos were selected that matched the requirement for all key joint points to be visible during the movements in each frame. Within the video clips, no changes or cuts were made. The total duration of the videos selected for analysis was 24 minutes 9 seconds, with an average duration of 2 minutes 25 seconds per video. The movements were categorized by the PanopticStudio Toolbox as a range of motion.

\section{Lindera Pose Estimation}

The Lindera-v2 algorithm is a combination of a 2D and 3D skeleton-based pose estimation. For this study, we needed the output of the 2D skeleton estimator to calculate 2D joint angles $[23,24]$. The 2D skeleton detector module of the Lindera-v2 algorithm is based on the tf-pose-estimation repository [25]. This repository is a TensorFlow implementation of various deep learning models [13] that represent human pose estimation models based on convolutional pose machines [26]. The original repository also provides some model variants that run on mobile devices. The short version of the repository used with the Lindera-v2 algorithm has also implemented the additional Openpose Body 25 model, which provides 25 body joint coordinates for each input image ( $x$ in the direction of the image width and $y$ in the direction of the image height) instead of 18 body joint coordinates, as was the case in the original repository model variants (Figure 1). The 2D module of the Lindera-v2 
algorithm produced a coordinate-time list for each joint and each frame. These time series data were then used to geometrically calculate the corresponding 2D joint angles for each frame of the processed input video. To smooth the angle-time series data, we used digital filtering with a size 11 Bartlett window.

Figure 1. Two-dimensional pose estimation by skeleton fitting, based on 25 body joint coordinates.
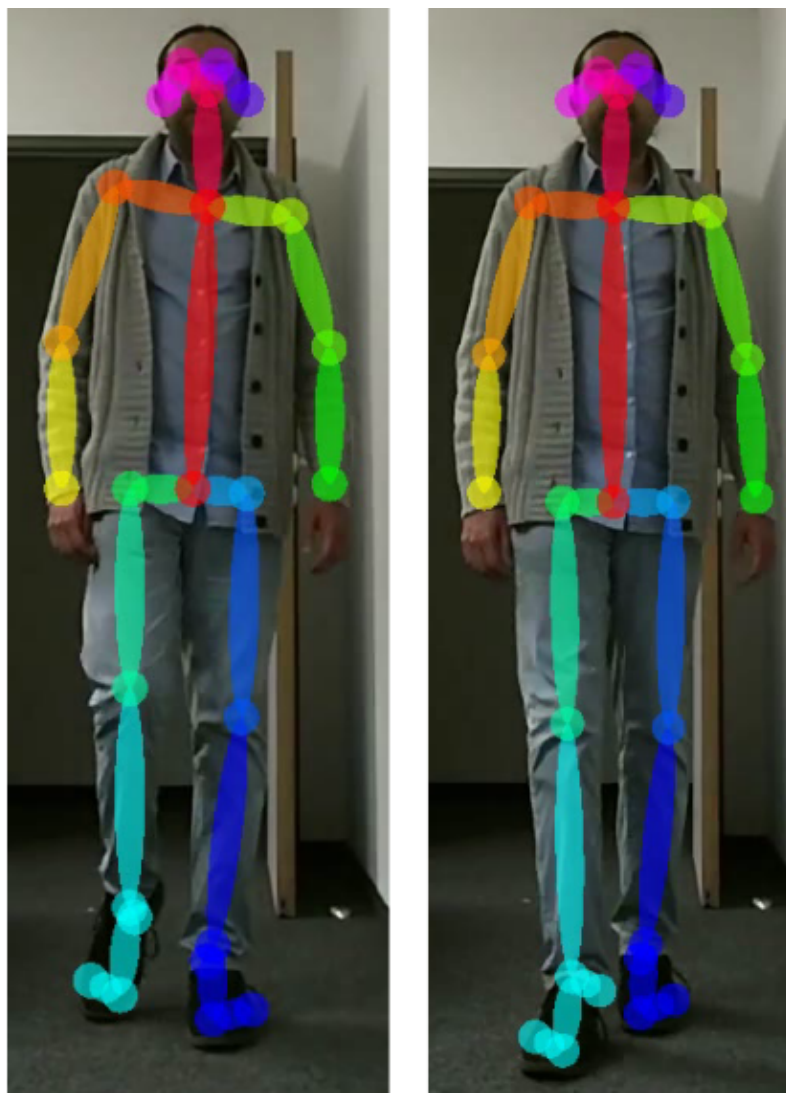

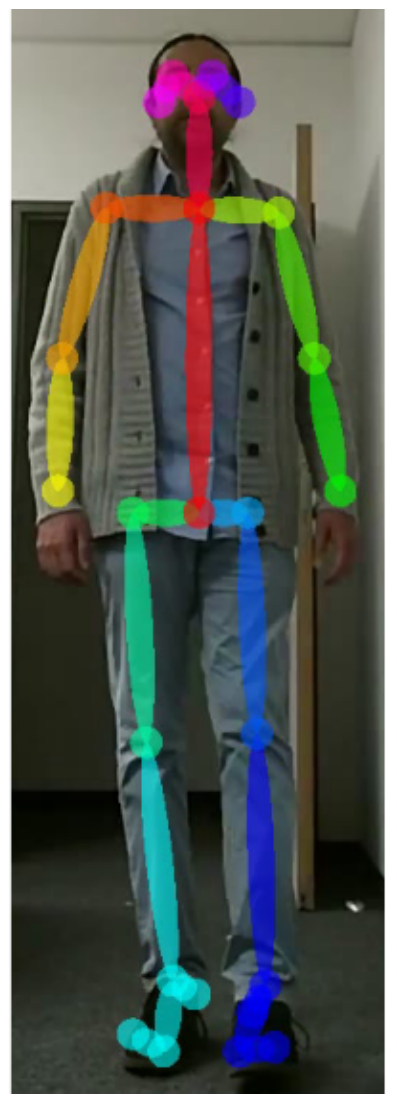

\section{Reference Standard}

The Panoptic Studio data set from Carnegie Mellon University $[21,22]$ is a data set shared for research purposes. Unlike the Lindera app, the PanopticStudio Toolbox is not a monocular $2 \mathrm{D}$ estimator but rather a massive multiview system consisting of the following: (1) $480 \mathrm{VGA}$ cameras with a resolution of 640 $\times 480$ pixels and 25 frames per second (fps); (2) 31 HD cameras with a resolution of $1920 \times 1080$ pixels and $30 \mathrm{fps}$; (3) 10 Kinect II sensors of $1920 \times 1080$ pixels (RGB), $512 \times 424$ pixels (depth), and $30 \mathrm{fps}$; and (4) 5 digital light processing projectors.

The 2D skeleton of the 2D Panoptic Studio pose detector has 15 anatomical landmarks. The $2 \mathrm{D}$ detector uses appearance information in the interpretation and includes connectivity information.

\section{Statistical Analysis}

The value tables for the respective joint angles were clustered, and missing values were imputed using a simple moving average. The mean difference (bias) between the Lindera-v2 algorithm estimates and the reference standard values was calculated for each joint. Furthermore, the mean absolute error, the root mean squared error, and the symmetric mean absolute percent error of the 2D angles were used. The intraclass correlation coefficient (ICC[A,2]) was calculated for the data using the 2-way mixed-effects model as a measure of agreement between the 2 measurement methods. An ICC in the range of
0 indicates random evaluation behavior, and a value of 1 is regarded as an ideal reliable feature evaluation by the evaluators. We used the definition in which values greater than 0.7 are generally regarded as indicators of good agreement [27]. Values up to approximately 0.3 are regarded as a low correlation, and those of approximately 0.5 or more are regarded as a medium correlation. A further classification according to Fleiss [28] was used to assesses the ICC classification, with 0.00 to 0.40 indicating poor agreement, 0.40 to 0.75 indicating fair to good agreement, and 0.75 to 1.00 indicating excellent agreement.

A cross-correlation was calculated for the time series to verify whether there was a temporal shift in the data. To verify the stationarity of the data, which is a prerequisite for cross-correlation testing, we used the augmented Dickey-Fuller test. The data were first evaluated in IBM SPSS Statistics (version 25.0; IBM Corp) and then in the programming language $\mathrm{R}$ in RStudio (version 3.5.1; RStudio Inc).

\section{Results}

In order to evaluate the accuracy of the movement signals recorded, we analyzed a total of 30,000 data pairs for each joint, comparing the joint angles obtained using the Lindera-v2 algorithm with those of the PanopticStudio Toolbox data set (the reference standard). Table 1 displays the 10 joints analyzed; the key points used for calculating the joint angles; the average difference (bias) between the estimated values of the Lindera-v2 
algorithm and the reference standard; and the mean absolute error (MAE), mean absolute deviation, root mean square error (RMSE), symmetric mean absolute percentage error (sMAPE), and ICC. The angles used were determined trigonometrically for both measuring methods.

Table 1. Mean angle difference and ICC of Lindera-v2 and the Panoptic Studio data set for the joints analyzed.

\begin{tabular}{|c|c|c|c|c|c|c|c|c|}
\hline Joint & $\begin{array}{l}2 \mathrm{D}^{\mathrm{a}} \text { key points } \\
\text { used }\end{array}$ & $\begin{array}{l}\text { Difference in 2D an- } \\
\text { gles }\left({ }^{\circ}\right) \text {, mean }(\mathrm{SD}) \text {; } \\
95 \% \mathrm{CI}\end{array}$ & $\begin{array}{l}\text { MAE }^{\mathrm{b}} \text { of } 2 \mathrm{D} \\
\text { angles }\left(^{\circ}\right)\end{array}$ & $\operatorname{MAD}^{c}\left({ }^{\circ}\right)$ & $\begin{array}{l}\operatorname{RMSE}^{\mathrm{d}} \text { of } 2 \mathrm{D} \\
\text { angles }\left(^{\circ}\right)\end{array}$ & $\begin{array}{l}\text { sMAPE }^{\mathrm{e}} \\
(\%)\end{array}$ & $\operatorname{ICC}^{\mathrm{f}}(95 \% \mathrm{CI})$ & $\begin{array}{l}\text { SE of mean } \\
\text { difference }\end{array}$ \\
\hline Right shoulder & $\begin{array}{l}\text { Right hip, } \\
\text { shoulder, and } \\
\text { elbow }\end{array}$ & $\begin{array}{l}2.71(10.28) \\
2.59 \text { to } 2.83\end{array}$ & 6.48 & 4.10 & 10.63 & 23.33 & $\begin{array}{l}0.978 \\
(0.973 \text { to } 0.981)\end{array}$ & 0.06 \\
\hline Left shoulder & $\begin{array}{l}\text { Left hip, shoul- } \\
\text { der, and elbow }\end{array}$ & $\begin{array}{l}-0.07(12.11) \\
-0.21 \text { to } 0.07\end{array}$ & 3.98 & 3.20 & 12.12 & 10.71 & $\begin{array}{l}0.951 \\
(0.950 \text { to } 0.952)\end{array}$ & 0.07 \\
\hline Right elbow & $\begin{array}{l}\text { Right shoulder, } \\
\text { elbow, and } \\
\text { wrist }\end{array}$ & $\begin{array}{l}-1.01(12.12) \\
-1.15 \text { to }-0.87\end{array}$ & 6.18 & 4.30 & 12.16 & 6.64 & $\begin{array}{l}0.983 \\
(0.983 \text { to } 0.984)\end{array}$ & 0.07 \\
\hline Left elbow & $\begin{array}{l}\text { Left shoulder, } \\
\text { elbow, and } \\
\text { wrist }\end{array}$ & $\begin{array}{l}0.24(6.20) \\
0.17 \text { to } 0.31\end{array}$ & 3.15 & 2.84 & 6.21 & 9.17 & $\begin{array}{l}0.997 \\
(0.997 \text { to } 0.997)\end{array}$ & 0.04 \\
\hline Right hip & $\begin{array}{l}\text { Right shoulder, } \\
\text { hip, and knee }\end{array}$ & $\begin{array}{l}-0.05(6.06) \\
-0.12 \text { to } 0.02\end{array}$ & 4.45 & 4.68 & 6.06 & 3.01 & $\begin{array}{l}0.983 \\
(0.983 \text { to } 0.983)\end{array}$ & 0.04 \\
\hline Left hip & $\begin{array}{l}\text { Left shoulder, } \\
\text { hip, and knee }\end{array}$ & $\begin{array}{l}-0.61(3.85) \\
-0.66 \text { to }-0.57\end{array}$ & 2.29 & 2.29 & 3.90 & 1.74 & $\begin{array}{l}0.992 \\
(0.992 \text { to } 0.993)\end{array}$ & 0.02 \\
\hline Right knee & $\begin{array}{l}\text { Right hip, knee, } \\
\text { and ankle }\end{array}$ & $\begin{array}{l}-1.37(2.97) ; \\
-1.40 \text { to }-1.34\end{array}$ & 2.58 & 2.93 & 3.27 & 1.56 & $\begin{array}{l}0.985 \\
(0.974 \text { to } 0.990)\end{array}$ & 0.02 \\
\hline Left knee & $\begin{array}{l}\text { Left hip, knee, } \\
\text { and ankle }\end{array}$ & $\begin{array}{l}0.84(4.31) \\
0.79 \text { to } 0.89\end{array}$ & 2.28 & 2.45 & 4.44 & 1.39 & $\begin{array}{l}0.971 \\
(0.968 \text { to } 0.974)\end{array}$ & 0.03 \\
\hline Neck & $\begin{array}{l}\text { Pelvis, neck, } \\
\text { and head }\end{array}$ & $\begin{array}{l}-3.07(6.43) ; \\
-3.14 \text { to }-2.99\end{array}$ & 4.47 & 3.63 & 7.13 & 3.20 & $\begin{array}{l}0.951 \\
(0.914 \text { to } 0.969)\end{array}$ & 0.04 \\
\hline Pelvis & $\begin{array}{l}\text { Left knee, } \\
\text { pelvis, and right } \\
\text { knee }\end{array}$ & $\begin{array}{l}0.15(2.03) \\
0.14 \text { to } 0.18\end{array}$ & 1.40 & 1.64 & 2.04 & 5.42 & $\begin{array}{l}0.996 \\
(0.996 \text { to } 0.996)\end{array}$ & 0.01 \\
\hline
\end{tabular}

a 2 : two-dimensional.

${ }^{\mathrm{b}} \mathrm{MAE}$ : mean absolute error.

${ }^{\mathrm{c}}$ MAD: mean absolute deviation.

${ }^{\mathrm{d}} \mathrm{RMSE}$ : root mean square error.

e SMAPE: symmetric mean absolute percentage error.

${ }^{\mathrm{f}}$ ICC: intraclass correlation coefficient $\operatorname{ICC}(\mathrm{A}, 2)$.

The data collected indicated both a negative and a positive bias. The mean difference of the joint angles that was nearest to the baseline was identified in the right hip $\left(-0.05^{\circ}, \mathrm{SD} 6.06^{\circ}\right)$. The joint with the highest mean difference (ie, with the greatest difference from 0$)$ was the neck $\left(-3.07^{\circ}\right.$, SD $\left.6.43^{\circ}\right)$. The mean joint angle accuracy was used to show the average magnitude of the errors. The mean absolute error of the angle measurement closest to the baseline was observed in the pelvis $\left(1.40^{\circ}, \mathrm{SD}\right.$ $\left.1.48^{\circ}\right)$. In contrast, the highest mean absolute error was observed in the right shoulder $\left(6.48^{\circ}, \mathrm{SD} 8.43^{\circ}\right)$. The standard deviation was also lowest in the pelvis (SD $3.36^{\circ}$ ), and the highest standard deviation was found to be in the left shoulder (SD $11.45^{\circ}$ ). The root mean square error was also applied, although this tends to give weight to large errors. The RMSE indicated low accuracy in the right elbow $\left(12.16^{\circ}\right)$ and high accuracy in the pelvis $\left(2.04^{\circ}\right)$. Since the mean absolute percentage error cannot be used when values are 0 (as this would result in division by 0 ), we used the SMAPE, which was lowest in the left knee $(1.39 \%)$ and highest in the right shoulder (23.33\%).

The intraclass correlation coefficient for the joint angles is also shown in Table 1 and represents agreement between the 2 measurement methods (Lindera-v2 vs the PanopticStudio Toolbox). In accordance with the McGraw and Wong convention [29], the intraclass correlation coefficient $\operatorname{ICC}(\mathrm{A}, 2)$ was used (ie, a 2-way mixed type with average measures and absolute agreement). The highest ICC value was found in the left elbow joint (average measure of 0.997 , 95\% CI $0.997-0.997)$. In contrast, the lowest ICC values were in the neck (average measure of $0.951,95 \%$ CI 0.914-0.969).

Interpretation of the measurement values based on mean values can lead to biased findings (eg, in the case of extreme outliers). 
Since the median is less affected by outliers, we used box plots for the differences in joint angle values measured with the Lindera-v2 and the reference standard. Figure 2 shows a box-and-whisker plot without outliers to facilitate closer examination of the boxes. The ends of the whiskers represent $1.5 \times \mathrm{IQR}$. The median with the greatest difference in comparison with the 0 value was detected in the right shoulder $\left(3.17^{\circ}\right)$, and the joint angle median nearest to the baseline was the pelvis joint $\left(0.19^{\circ}\right)$. Figure 3 shows the box-and-whisker plot with outliers. The third quartile of the right shoulder was the farthest from the baseline of all the joints, with a value of $5.87^{\circ}$. The lowest first quartile was in the neck, measuring $-5.34^{\circ}$. The smallest IQR, ranging from $-0.81^{\circ}$ to $1.27^{\circ}$, was in the pelvis. The most extreme outliers in this plot were found in the right elbow, where the minimum was $-106.00^{\circ}$ and the maximum was $125.71^{\circ}$. However, the outlier with the greatest difference in comparison to 0 was in the left shoulder, with a difference of $157.10^{\circ}$.

Figure 2. Box plot showing differences in the Lindera-v2 and reference standard values, measured across all joints tested.

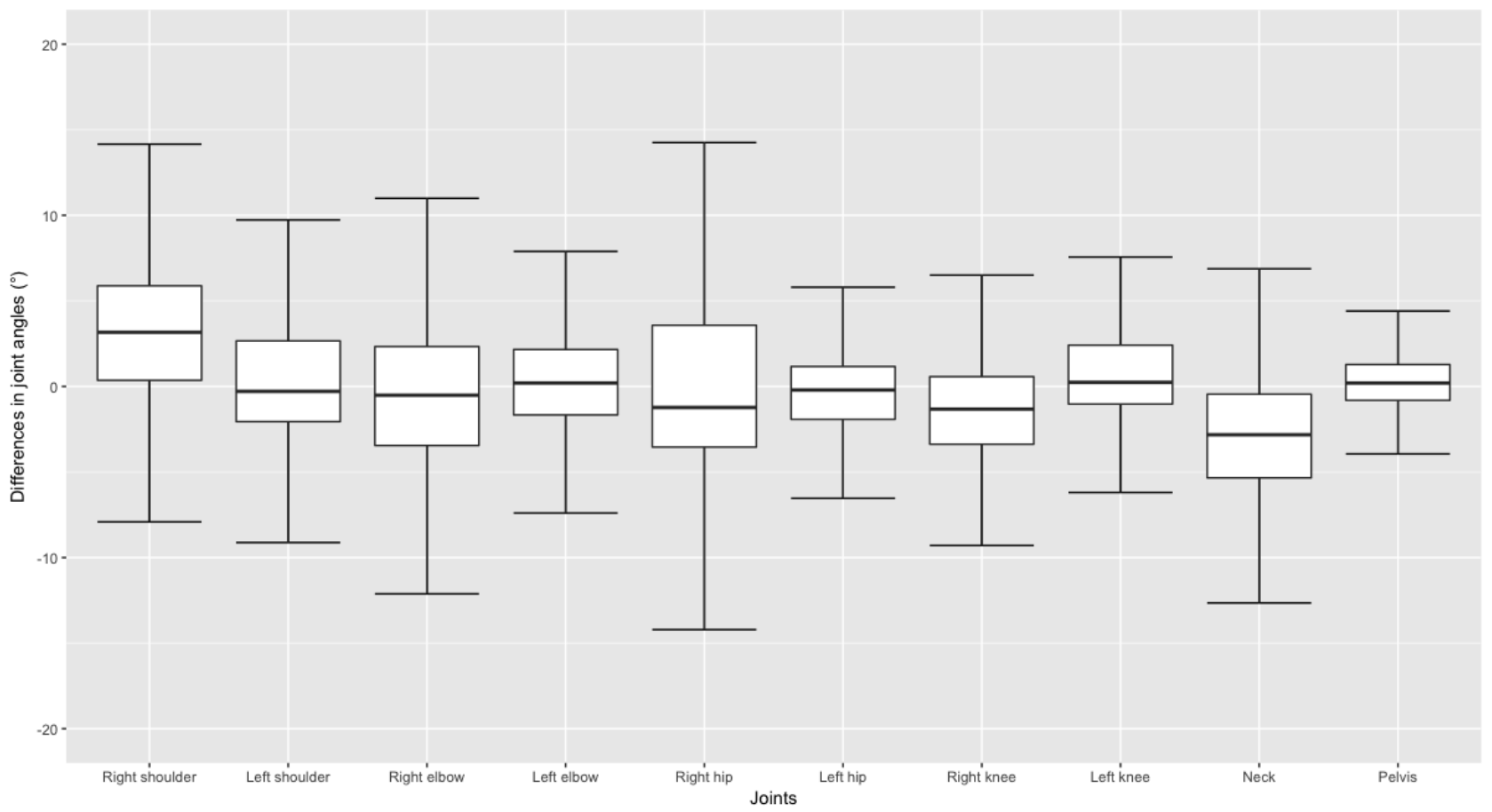

Figure 3. Box plot with outliers showing differences in the Lindera-v2 and reference standard values, measured across all joints tested.

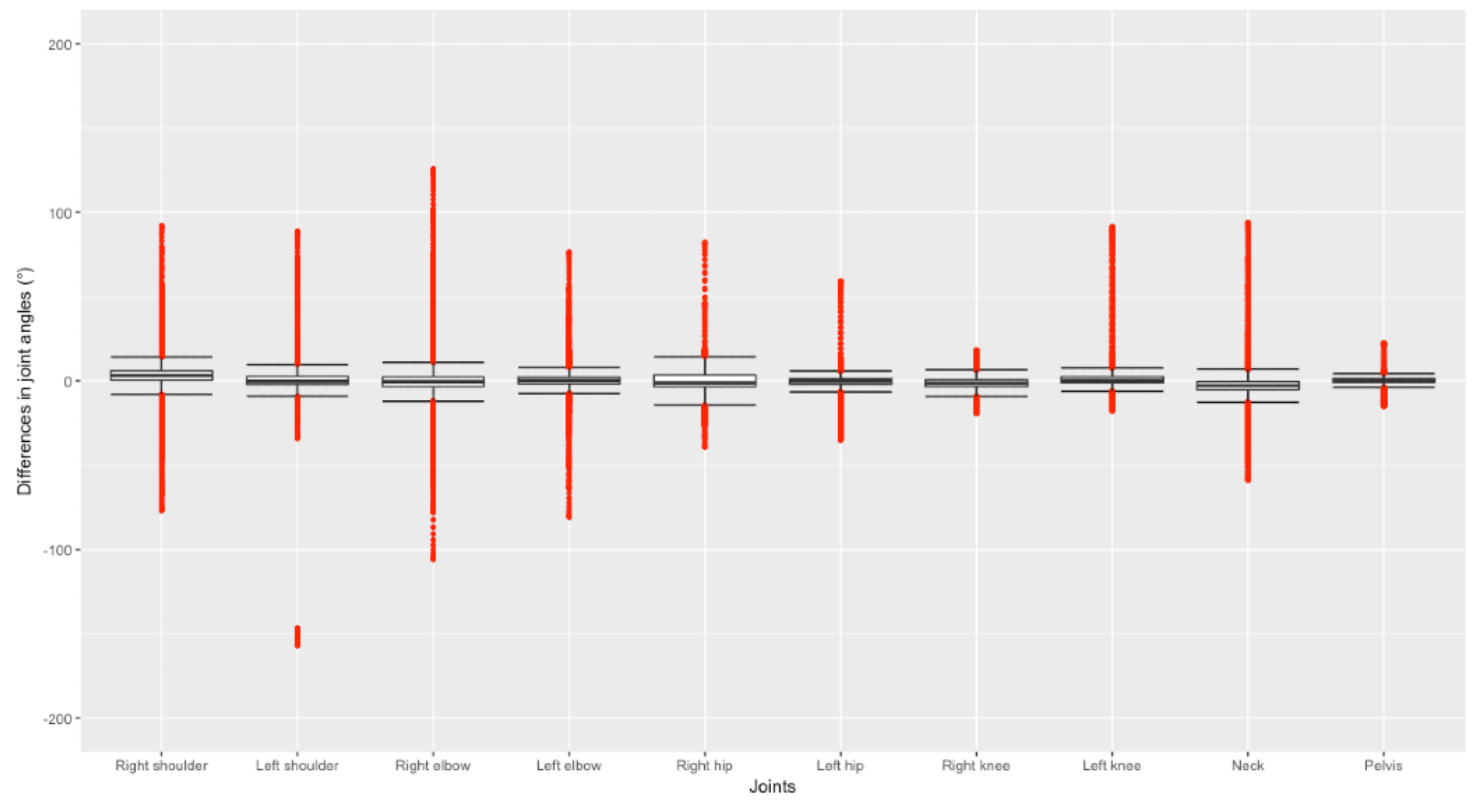

To examine the ICC more closely and analyze the potential influence of single videos on the ICC values of the joints, our next step entailed calculating an ICC for each video. Figure 4 shows a dot plot of the ICC for the 10 videos used for the accuracy measurement in each joint. For the neck joint, 5 videos had an ICC below 0.75 . However, there were no videos in which all joints had remarkably low ICC values. With the exception of the neck, all joints in all videos had an ICC value above 0.75 . 
The cross-correlation function was applied to the selected time series in order to examine the temporal lag. The results in Table 2 show that no time delays could be detected in the values measured. These would have been visible at an increased correlation at a time outside lag 0 . However, all graphs (Figure
5) showed the highest correlation at lag 0 . The dotted blue lines represent the confidence interval of the estimated correlation values. If a value was outside the range of the interval, the correlation was considered significant.

Figure 4. Dot plot of the intraclass correlation coefficient comparing Lindera-v2 and reference standard for the 10 single videos used for accuracy measurement in each joint.

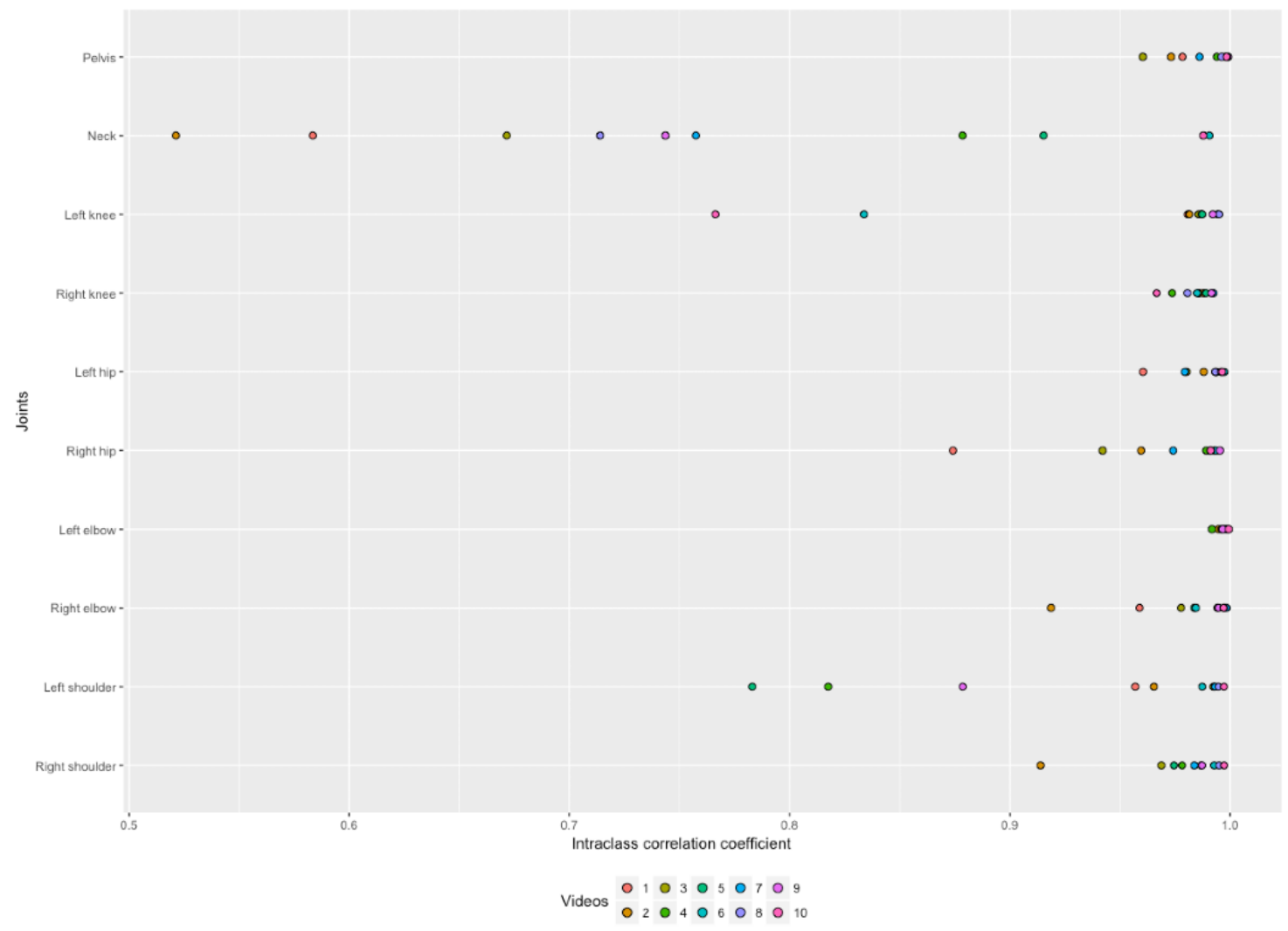


Figure 5. Cross-correlation graph of Lindera-v2 and Panoptic Studio data set values. One lag represents 1 sample (frame). ACF: autocorrelation function.

Right shoulder

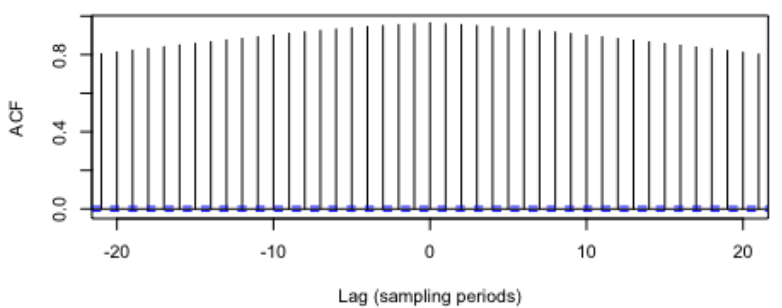

Right elbow

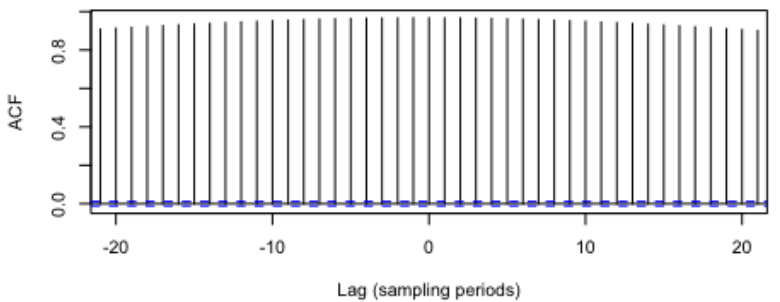

Right hip

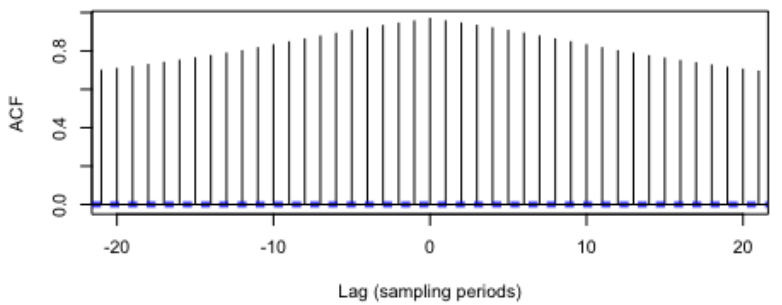

Right knee

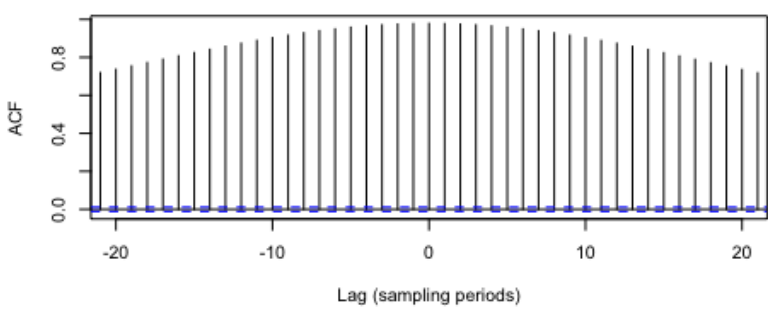

Neck

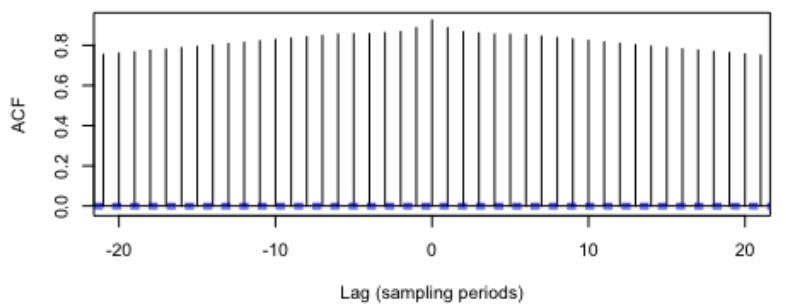

Left shoulder

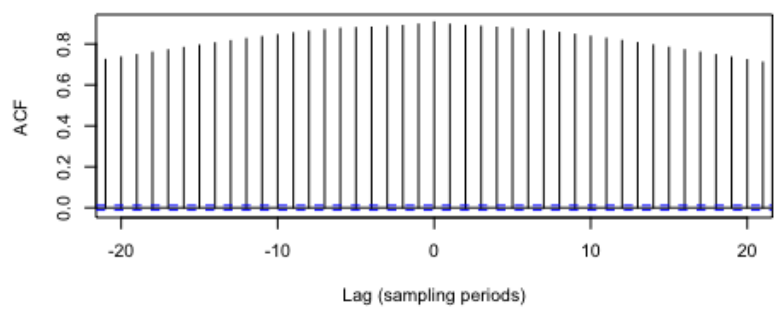

Left elbow

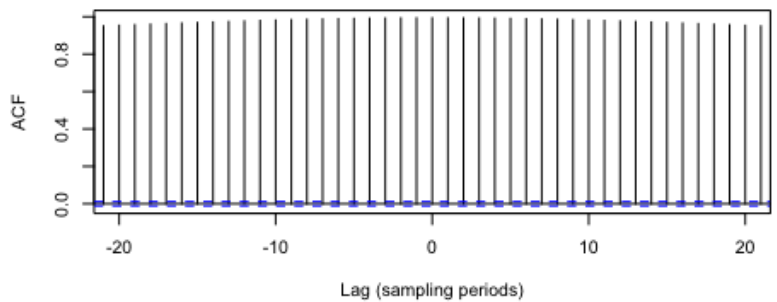

Left hip

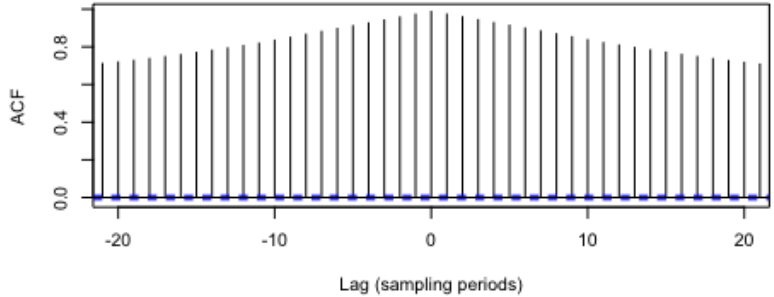

Left knee

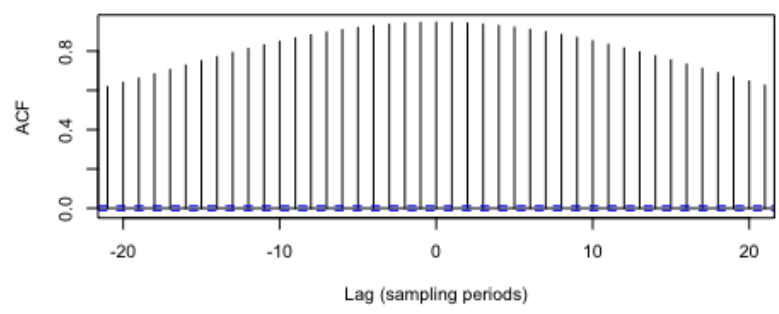

Pelvis

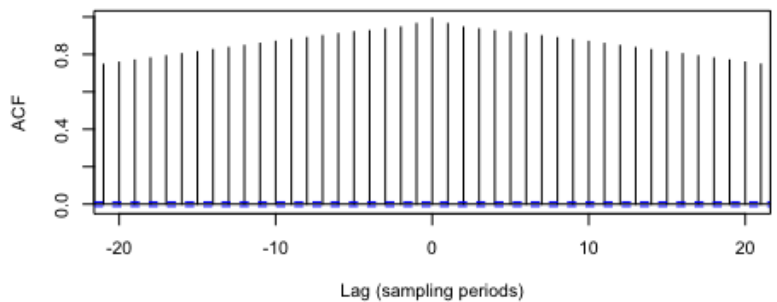


Table 2. Maximum cross-correlations of Lindera-v2 and reference standard values.

\begin{tabular}{lll}
\hline Joint & Lag value with maximum correlation & Maximum correlation coefficient \\
\hline Right shoulder & 0 & 0.96 \\
Left shoulder & 0 & 0.91 \\
Right elbow & 0 & 0.97 \\
Left elbow & 0 & 0.99 \\
Right hip & 0 & 0.97 \\
Left hip & 0 & 0.99 \\
Right knee & 0 & 0.98 \\
Left knee & 0 & 0.95 \\
Neck & 0 & 0.92 \\
Pelvis & 0 & 0.99 \\
\hline
\end{tabular}

\section{Discussion}

\section{Principal Results}

The goal of this study was to validate the accuracy of the 2D pose estimation of joint angles obtained from the Lindera-v2 algorithm, using the PanopticStudio Toolbox, which served as the reference standard. Therefore, we analyzed 30,000 data pairs for each joint angle during diverse total body motion activity. First, the mean difference and error measures were compared for each joint. Second, the ICC was compared for each joint. In order to verify agreement between the 2 measurement methods (the Lindera-v2 and the PanopticStudio Toolbox data set), we analyzed the ICC values for each of the 10 videos. Finally, we examined the potential temporal lag through cross-correlation. The results of the study indicate that the $2 \mathrm{D}$ pose estimation method used had excellent agreement with the reference standard. Furthermore, the Lindera-v2 algorithm had no temporal lag.

The mean angle generated for the right hip by the Lindera-v2 algorithm was the closest to the reference standard. Even the value with the greatest difference from 0 (found in the neck) was acceptable. However, these values should be treated with caution because mean values can lead to biased results. Therefore, we displayed the median values in box plots. The medians of all joints compared with the reference standard ranged from a difference of $0.19^{\circ}$ (pelvis joint) to $3.17^{\circ}$ (right shoulder). In all joints, the IQR was within $6^{\circ}$ and $-6^{\circ}$, which means that $50 \%$ of the values were within this range. These acquired values provide a promising starting point upon which to base mobility assessments and 3D pose estimation. A further reason why box plots were used was to identify outliers because the RMSE used gives greater weight to large errors. Figure 3 shows that in the box-and-whisker plots, the data recording the difference between the Lindera-v2 algorithm and the reference standard had few very high outliers. In the right elbow, for example, the outliers were particularly high compared with the other joints. Since the RMSE squares the errors before averaging, the RMSE in this joint was the highest. Since large outliers can be quickly identified as such by an experienced user, the weighting of large outliers by the RMSE does not seem appropriate. Hence, the MAE might be the more appropriate measure. The sMAPE is more resistant to outliers due to defined error limits because it gives less weight to outliers than other measures that have no error limits; it was applied additionally for this reason [30]. An advantage over the mean absolute percentage error is that the sMAPE cannot be extremely large or infinite [30]. The sMAPE in our evaluation was particularly high in the right shoulder (23.33\%). Possible joint "losses" could be an explanation due to bad visibility of the joints in the videos, perhaps due to the clothing of the participant or the lighting used.

The ICC agreement between the 2 measurement methods can be interpreted as excellent (according to the classification presented by Fleiss [28]). All joints had an ICC value of at least 0.951 . Furthermore, the $95 \%$ confidence interval of the ICC for all joints can be classified as excellent. Our analysis of the individual videos showed why the neck had the lowest overall agreement in comparison with the other joints. In several videos, the agreement could be interpreted as fair to good. We assume that this relates to the approximations of neck positions, since these were calculated from the key point of the nose in the Lindera algorithm. By applying cross-correlation, angles estimated through the Lindera-v2 algorithm showed no temporal lag.

Early research and reviews published in 2016 reported that the Kinect skeleton-tracking algorithm indicated poor validity and large errors with respect to most kinematic variables [7]. Clark et al [10] recommended that the Kinect system be carefully chosen for specific use cases (eg, trunk angles can be highly accurate). An extensive recent review by Poitras et al [31] on the validity and reliability of wearable sensors for joint angle estimation revealed mixed results. The results presented in this study are therefore very promising, not only because of the acceptable accuracy of the angles but also because the usability of smartphone apps (compared with the Kinect system or wearable sensors) offers major advantages. Schurr et al [32] showed a moderate to strong relationship between a $2 \mathrm{D}$ video camera and 3D motion capture analyses. From this point of view, 2D pose estimations are applicable in clinical practice. Even though 2D cameras offer clinicians a valuable kinematic measurement tool, the use of a smartphone would be far less 
complicated and would make the technology available to a wide user group.

Valid and reliable 2D joint angles are an important first step on the way to valid and reliable 3D joint angles. Therefore, in the next step, the 2D data from the evaluation will be transformed into 3D pose estimation angles using deep convolutional neural networks. A validation of the $3 \mathrm{D}$ joint angle accuracy of the resulting data will show whether the requirements for clinical practice are met.

\section{Limitations}

Although this study showed excellent agreement to a reference standard, a validity study using a state-of-the-art marker-based motion capture system as a ground truth is necessary for a thorough validation. The comparison to the reference standard is an important step toward accuracy assurance but does not replace a proof of validity.

To determine a systematic error in the algorithm by an offset, a static setup would be needed. From this, a Euclidean distance could be calculated to identify a precise source of error. The mean joint position error is the most frequently used method for verification of the accuracy of a pose estimation. However, since determination of the coordinates in millimeters in space was not possible in these data sets, accuracy verification was carried out for the joint angles. Verification of the precision showing the repeatability of the data was not planned in this project, since measurement using the Lindera-v2 was carried out once and the movements were not repeated in a standardized manner. However, the precision of the time stamps within the measurement of the evaluated movements can be seen from the standard errors of the mean difference. A validation of the precision will be the subject of further studies.

\section{Perspectives}

In geriatrics, orthopedics, and neurology in particular, accurate and validated mobility analyses such as the Lindera-v2 could help medical professionals confirm diagnoses and track the success of treatments. Mobility assessments have very high relevance for a multitude of clinical uses (eg, older adults and patients with more severe diseases who have a higher risk of falling) [33,34]; in this case, fall risk assessments could be of high value. Assessment of kinematic variables, such as specific joint angles, can be accessed via 2D skeleton data if viewed from specific angles, and such data can also be used for rehabilitative purposes in physical therapy or sports science. Furthermore, the $2 \mathrm{D}$ values analyzed in this study constitute an encouraging basis for $3 \mathrm{D}$ pose estimation, which will be the next step in accuracy validation.

\section{Conclusions}

The results of the study indicate that $2 \mathrm{D}$ pose estimation by means of a camera-based smartphone app can have excellent agreement with a validated reference standard. Furthermore, the Lindera-v2 algorithm was found to have no temporal lag. An assessment of kinematic variables, such as specific joint angles, can be performed with the algorithm, and these data showed only minimal deviations compared with data from a massive multiview system. In future studies, it will be important to test the app in a clinical context with participants with physical limitations.

\section{Acknowledgments}

We would like to thank Lindera GmbH for providing the raw data for the study. We acknowledge support from the German Research Foundation and the Open Access Publication Fund of Charité - Universitätsmedizin Berlin.

\section{Conflicts of Interest}

None declared.

\section{References}

1. Colyer SL, Evans M, Cosker DP, Salo AIT. A Review of the Evolution of Vision-Based Motion Analysis and the Integration of Advanced Computer Vision Methods Towards Developing a Markerless System. Sports Med Open 2018 Jun 05;4(1):24 [FREE Full text] [doi: 10.1186/s40798-018-0139-y] [Medline: 29869300]

2. van der Kruk E, Reijne MM. Accuracy of human motion capture systems for sport applications; state-of-the-art review. Eur J Sport Sci 2018 Jul 09;18(6):806-819. [doi: 10.1080/17461391.2018.1463397] [Medline: 29741985]

3. Merriaux P, Dupuis Y, Boutteau R, Vasseur P, Savatier X. A Study of Vicon System Positioning Performance. Sensors (Basel) 2017 Jul 07;17(7) [FREE Full text] [doi: 10.3390/s17071591] [Medline: 28686213]

4. Corazza S, Mündermann L, Gambaretto E, Ferrigno G, Andriacchi TP. Markerless Motion Capture through Visual Hull, Articulated ICP and Subject Specific Model Generation. Int J Comput Vis 2009 Sep 2;87(1-2):156-169. [doi: 10.1007/s11263-009-0284-3]

5. Leardini A, Chiari L, Della Croce U, Cappozzo A. Human movement analysis using stereophotogrammetry. Part 3. Soft tissue artifact assessment and compensation. Gait Posture 2005 Feb;21(2):212-225. [doi: 10.1016/j.gaitpost.2004.05.002] [Medline: $\underline{15639400]}$

6. Otte K, Kayser B, Mansow-Model S, Verrel J, Paul F, Brandt AU, et al. Accuracy and Reliability of the Kinect Version 2 for Clinical Measurement of Motor Function. PLoS One 2016 Nov 18;11(11):e0166532 [FREE Full text] [doi: 10.1371/journal.pone.0166532] [Medline: 27861541]

7. Springer S, Yogev Seligmann G. Validity of the Kinect for Gait Assessment: A Focused Review. Sensors (Basel) 2016 Feb 04;16(2):194 [FREE Full text] [doi: 10.3390/s 16020194] [Medline: 26861323] 
8. Bonnechère B, Jansen B, Salvia P, Bouzahouene H, Omelina L, Moiseev F, et al. Validity and reliability of the Kinect within functional assessment activities: comparison with standard stereophotogrammetry. Gait Posture 2014;39(1):593-598. [doi: 10.1016/j.gaitpost.2013.09.018] [Medline: 24269523]

9. Mentiplay BF, Perraton LG, Bower KJ, Pua Y, McGaw R, Heywood S, et al. Gait assessment using the Microsoft Xbox One Kinect: Concurrent validity and inter-day reliability of spatiotemporal and kinematic variables. J Biomech $2015 \mathrm{Jul}$ 16;48(10):2166-2170. [doi: 10.1016/j.jbiomech.2015.05.021]] [Medline: 26065332]

10. Clark RA, Mentiplay BF, Hough E, Pua YH. Three-dimensional cameras and skeleton pose tracking for physical function assessment: A review of uses, validity, current developments and Kinect alternatives. Gait Posture 2019 Feb;68:193-200. [doi: 10.1016/j.gaitpost.2018.11.029] [Medline: 30500731]

11. Fernandez-Baena A, Susin A, Lligadas X. Biomechanical Validation of Upper-Body and Lower-Body Joint Movements of Kinect Motion Capture Data for Rehabilitation Treatments. 2012 Presented at: 2012 Fourth International Conference on Intelligent Networking and Collaborative Systems; Sep 19-21, 2012; Bucharest, Romania. [doi: 10.1109/incos.2012.66]

12. Brau E, Jiang H. 3D Human Pose Estimation via Deep Learning from 2D Annotations. 2016 Presented at: Fourth International Conference on 3D Vision (3DV); Oct 25-28, 2016; Stanford, CA. [doi: 10.1109/3dv.2016.84]

13. Cao Z, Hidalgo G, Simon T, Wei S, Sheikh Y. OpenPose: Realtime Multi-Person 2D Pose Estimation using Part Affinity Fields. arXiv. 2019 May 30. URL: http://arxiv.org/abs/1812.08008 [accessed 2019-11-10]

14. Bogo F, Kanazawa A, Lassner C, Gehler P, Romero J, Black M. Keep It SMPL: Automatic Estimation of 3D Human Pose and Shape from a Single Image. In: Leibe B, Matas J, Sebe N, Welling M, editors. Computer Vision ECCV 2016. Cham, Switzerland: Springer International Publishing; 2016:561-578.

15. Liu S, Li Y, Hua G. Human Pose Estimation in Video via Structured Space Learning and Halfway Temporal Evaluation. IEEE Trans Circuits Syst Video Technol 2019 Jul;29(7):2029-2038. [doi: 10.1109/tcsvt.2018.2858828]

16. Moder M, Schellenbach M. Deep Learning Approach for Estimating a Human Pose on a Mobile Device. In: Kameas A, Stathis K, editors. Ambient Intelligence. Cham, Switzerland: Springer International Publishing; 2018:100-105.

17. Mehta D, Sridhar S, Sotnychenko O, Rhodin H, Shafiei M, Seidel H, et al. VNect. ACM Trans Graph 2017 Jul 20;36(4):1-14. [doi: 10.1145/3072959.3073596]

18. Qiang B, Zhang S, Zhan Y, Xie W, Zhao T. Improved Convolutional Pose Machines for Human Pose Estimation Using Image Sensor Data. Sensors (Basel) 2019 Feb 10;19(3):718 [FREE Full text] [doi: 10.3390/s19030718] [Medline: 30744191$]$

19. Hidalgo G, Raaj Y, Idrees H, Xiang D, Joo H, Simon T, et al. Single-Network Whole-Body Pose Estimation. 2019 Presented at: 2019 International Conference on Computer Vision (ICCV); Oct 27-Nov 2, 2019; Seoul, Korea. [doi: $10.1109 /$ iccv.2019.00708]

20. Huber S, Priebe JA, Baumann K, Plidschun A, Schiessl C, Tölle TR. Treatment of Low Back Pain with a Digital Multidisciplinary Pain Treatment App: Short-Term Results. JMIR Rehabil Assist Technol 2017 Dec 04;4(2):e11 [FREE Full text] [doi: 10.2196/rehab.9032] [Medline: 29203460]

21. Joo H, Simon T, Li X, Liu H, Tan L, Gui L, et al. Panoptic Studio: A Massively Multiview System for Social Interaction Capture. IEEE Trans Pattern Anal Mach Intell 2019 Jan 1;41(1):190-204. [doi: 10.1109/tpami.2017.2782743]

22. Simon T, Joo H, Matthews I, Sheikh Y. Hand Keypoint Detection in Single Images using Multiview Bootstrapping. arXiv. 2017 Apr 25. URL: http://arxiv.org/abs/1704.07809 [accessed 2019-11-11]

23. Azhand D, Rabe D, Müller D, Sattler I, Steinert D. Algorithm Based on One Monocular Video Delivers Highly Valid and Reliable Gait Parameters. arXiv. URL: http://arxiv.org/abs/2008.08045 [accessed 2020-09-08]

24. Rabe S, Azhand A, Pommer W, Müller S, Steinert A. Descriptive Evaluation and Accuracy of a Mobile App to Assess Fall Risk in Seniors: Retrospective Case-Control Study. JMIR Aging 2020 Feb 14;3(1):e16131 [FREE Full text] [doi: 10.2196/16131] [Medline: 32130111]

25. Kim I. Deep Pose Estimation implemented using Tensorflow with Custom Architectures for fast inference. GitHub. URL: https://github.com/ildoonet/tf-pose-estimation [accessed 2020-12-12]

26. Wei S, Ramakrishna V, Kanade T, Sheikh Y. Convolutional Pose Machines. 2016 Presented at: IEEE Conference on Computer Vision and Pattern Recognition (CVPR); June 27-30, 2016; Las Vegas, NV. [doi: 10.1109/cvpr.2016.511]

27. Wirtz M, Caspar F. Beurteilerübereinstimmung und Beurteilerreliabilität: Methoden zur Bestimmung und Verbesserung der Zuverlässigkeit von Einschätzungen mittels Kategoriensystemen und Ratingskalen. Göttingen, Germany: Hogrefe Verlag; 2002:978-973.

28. Fleiss J. The Design and Analysis of Clinical Experiments. New York, NY: John Wiley \& Sons; 1986.

29. McGraw KO, Wong SP. Forming inferences about some intraclass correlation coefficients. Psychol Methods 1996;1(1):30-46. [doi: 10.1037/1082-989X.1.1.30]

30. Chen C, Twycross J, Garibaldi JM. A new accuracy measure based on bounded relative error for time series forecasting. PLoS One 2017 Mar 24;12(3):e0174202 [FREE Full text] [doi: 10.1371/journal.pone.0174202] [Medline: 28339480]

31. Poitras I, Dupuis F, Bielmann M, Campeau-Lecours A, Mercier C, Bouyer L, et al. Validity and Reliability of Wearable Sensors for Joint Angle Estimation: A Systematic Review. Sensors (Basel) 2019 Mar 31;19(7):1555 [FREE Full text] [doi: 10.3390/s19071555] [Medline: 30935116] 
32. Schurr S, Marshall AN, Resch JE, Saliba SA. Two-Dimensional Video Analysis Is Comparable To 3D Motion Capture In Lower Extremity Movement Assessment. Int J Sports Phys Ther 2017 Apr;12(2):163-172 [FREE Full text] [Medline: 28515970]

33. Jia H, Lubetkin EI, DeMichele K, Stark DS, Zack MM, Thompson WW. Prevalence, risk factors, and burden of disease for falls and balance or walking problems among older adults in the U.S. Prev Med 2019 Sep;126:105737. [doi: 10.1016/j.ypmed.2019.05.025] [Medline: 31150739]

34. Jacobi L, Petzold T, Hanel A, Albrecht M, Eberlein-Gonska M, Schmitt J. [Epidemiology and prediction of the risk of falling in patients in acute care settings: Analysis of routine data from a university hospital]. Z Evid Fortbild Qual Gesundhwes 2017 Feb;120:9-15. [doi: 10.1016/j.zefq.2016.12.006] [Medline: 28284369]

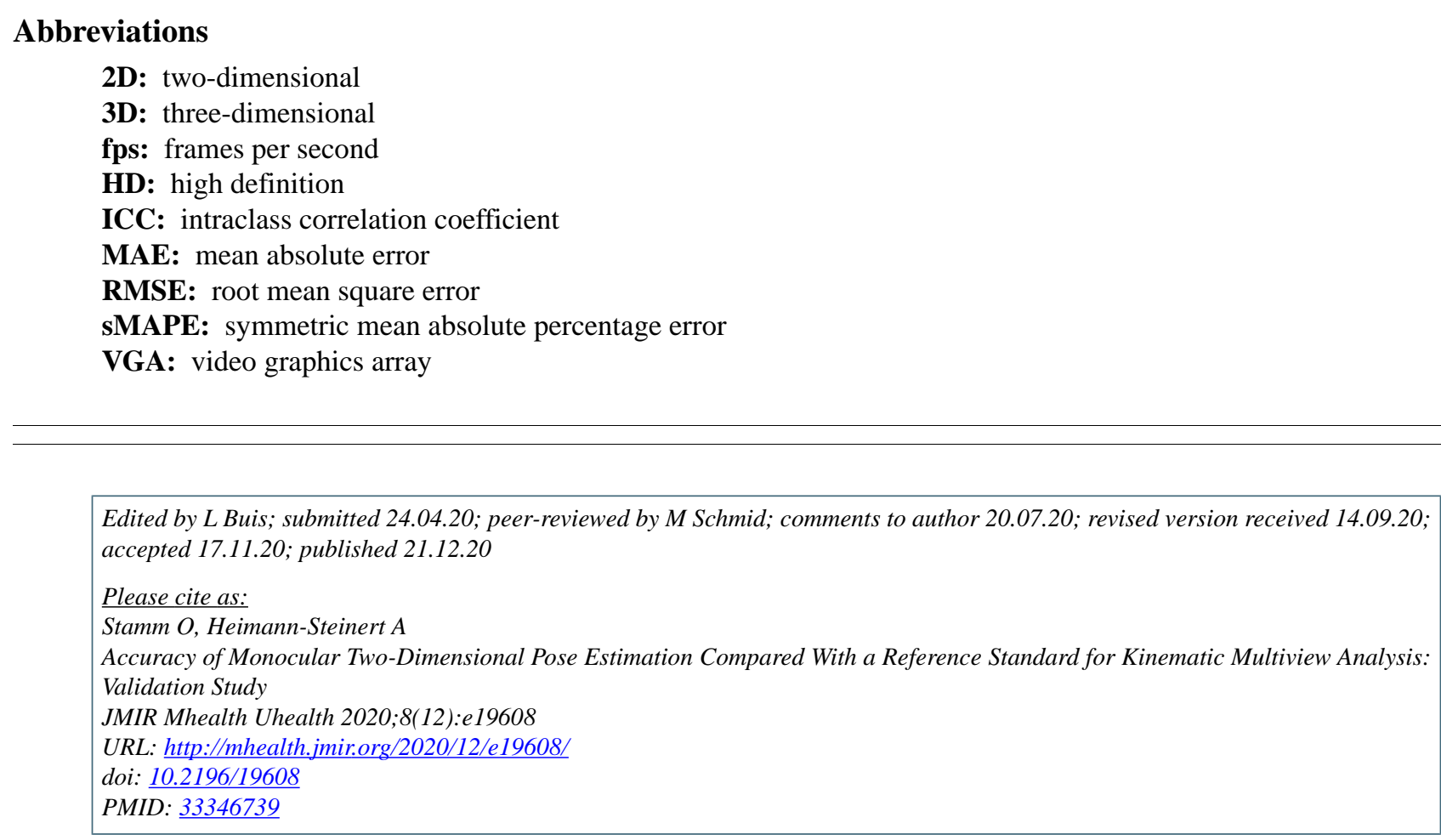

(C) Oskar Stamm, Anika Heimann-Steinert. Originally published in JMIR mHealth and uHealth (http://mhealth.jmir.org), 21.12.2020. This is an open-access article distributed under the terms of the Creative Commons Attribution License (https://creativecommons.org/licenses/by/4.0/), which permits unrestricted use, distribution, and reproduction in any medium, provided the original work, first published in JMIR mHealth and uHealth, is properly cited. The complete bibliographic information, a link to the original publication on http://mhealth.jmir.org/, as well as this copyright and license information must be included. 